\title{
Safety and efficacy of thoracoscopic sympathectomy for control of recurrent ventricular tachycardia in patients mainly with Chagas disease.
}

Rodrigo Kulchetscki ${ }^{1}$, Felipe Alexandre ${ }^{1}$, Marina Mayrink ${ }^{1}$, Carina Hardy ${ }^{2}$, Sissy de Melo ${ }^{1}$, Muhieddine Chokr ${ }^{1}$, Luis Abdalla ${ }^{1}$, Martino Martinelli Filho ${ }^{3}$, Francisco Darrieux ${ }^{4}$, Cristiano Pisani ${ }^{5}$, Mauricio Ibrahim Scanavacca ${ }^{6}$, and Paulo Pêgo-Fernandes ${ }^{7}$

${ }^{1}$ University of São Paulo Medical School

${ }^{2}$ Heart Institute

${ }^{3}$ Heart Institute (InCor) University of Sao Paulo Medical School

${ }^{4}$ University of São Paulo Medical School, Arrhythmia Unit São Paulo, SP, BR

${ }^{5}$ Heart Institute, University of São Paulo Medical School

${ }^{6}$ Cardiac Arrhythmias and Electrophysiology Unit, Heart Institute, University of Sao Paulo Medical School, São Paulo, Brazil

${ }^{7}$ University of Sao Paulo Heart Institute

April 28, 2021

\begin{abstract}
Introduction: The autonomous system plays an important role as a trigger of cardiac arrhythmias. Cardiac sympathetic denervation (CSD) achieved by stellate and proximal thoracic ganglia resection has been reported as an alternative approach for the management of ventricular arrhythmias (VA) in structural heart disease (SHD) patients. Insufficient data regarding Chagas Disease $(\mathrm{ChD})$ is available. Methods: Patients who underwent CSD for better management of ventricular arrhythmias (VA) in SHD, mainly $\mathrm{ChD}$, in a single tertiary center in Brazil were evaluated for safety and efficacy outcomes. Results: Between June 2014 and March 2020, fourteen patients (age 59 \pm 7.5 , 85\% male, mean ejection fraction $30.5 \pm 7.9 \%$ ) were submitted to left or bilateral CSD. In a median follow-up time of 143 (Q1: 30; Q3: 374) days, eight patients (57,2\%) presented VT recurrence. A significant reduction in the median burden of ventricular arrhythmias comparing six months before and after procedure (10 to $0 ; \mathrm{p}=0.004)$. For the nine $\mathrm{ChD}$ patients, the median burden of appropriate therapies was also reduced $(11$ to $0 ; \mathrm{p}=0.008)$. There were two cases of clinically relevant pneumothorax and three cases of transient hemodynamic instability, but no direct procedure-related deaths occurred. Additionally, there was no long-term adverse events, Conclusion: CSD is safe and seems to be effective in reducing the burden of VT/VT storm in SHD patients, including ChD patients. Randomized trials are needed to clarify its role in the management of these patients.
\end{abstract}

\section{Hosted file}

Paper_CSD_retrospective_ChD_only.pdf available at https://authorea.com/users/410715/articles/ 519990-safety-and-efficacy-of-thoracoscopic-sympathectomy-for-control-of-recurrentventricular-tachycardia-in-patients-mainly-with-chagas-disease 
Figure 1: Box Plot comparing the number of VT and/or appropriate therapies in the prior 6 months before and the follow-up period after the procedure. A - All population. B - Chagas Disease Only

A. All Population

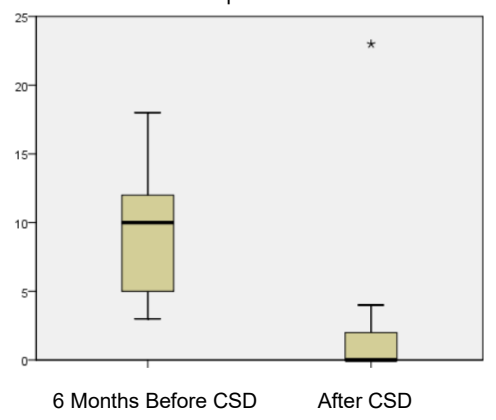

B. Chagas Disease Only

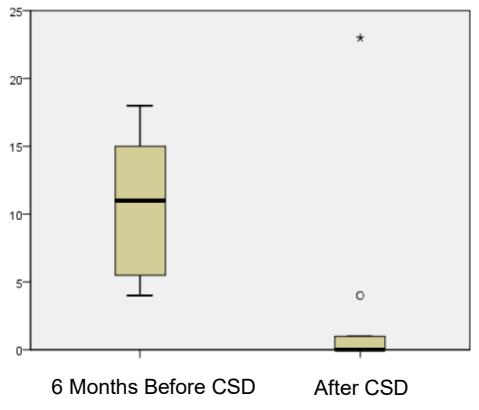


Figure 2: Kaplan-Meier curve of survival time to recurrence of ICD therapies following CSD.

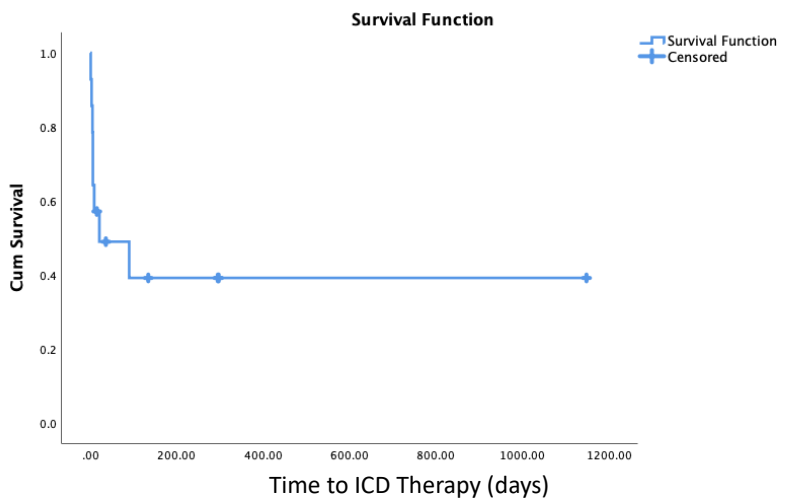


Figure 3: Plot showing the frequency of ICD therapies during the 6 months before (blue bars) and after (red bars) CSD for 11 patients with ICDs. Each bar represents an individual patient.

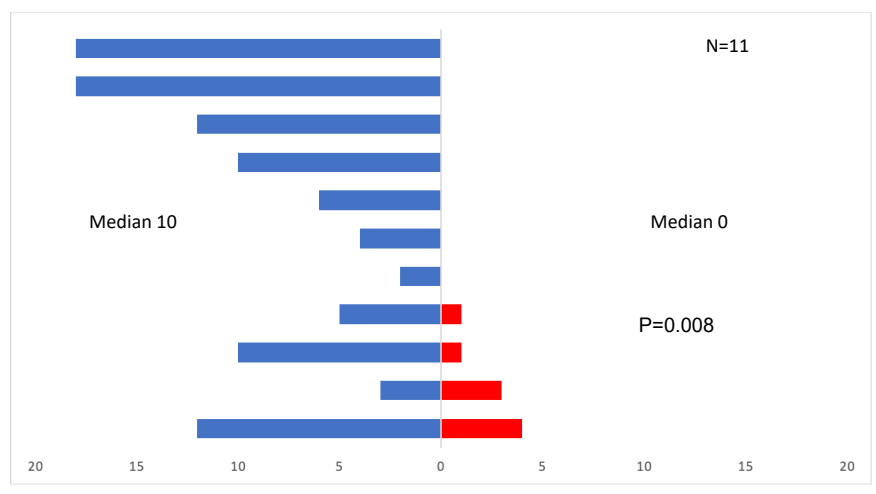


Figure 4: Kaplan-Meier curve of survival time to death of ICD therapies following CSD.

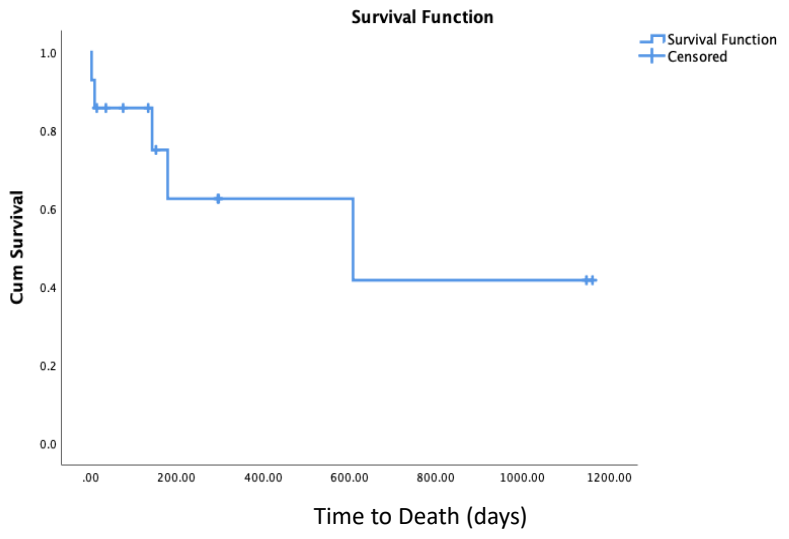

